\title{
Impact of COVID-19 Pandemic on the Incidence, Prehospital Evaluation, and Presentation of Ischemic Stroke at a Nonurban Comprehensive Stroke Center
}

\author{
Cesar Velasco, ${ }^{1}$ Brandon Wattai, ${ }^{2}$ Scott Buchle, ${ }^{2}$ Alicia Richardson, ${ }^{1,3}$ Varun Padmanaban, ${ }^{3}$ \\ Kathy J. Morrison, ${ }^{1}$ Raymond Reichwein, ${ }^{4,1}$ Ephraim Church, ${ }^{3}$ Scott D. Simon, ${ }^{3}$ \\ and Kevin M. Cockroft $\mathbb{D}^{3,1}$ \\ ${ }^{1}$ Penn State Comprehensive Stroke Center, Penn State Health, Hershey, PA, USA \\ ${ }^{2}$ Life Lion EMS, Penn State Health, Hershey, PA, USA \\ ${ }^{3}$ Department of Neurosurgery, Penn State Health, Hershey, PA, USA \\ ${ }^{4}$ Department of Neurology, Penn State Health, Hershey, PA, USA \\ Correspondence should be addressed to Kevin M. Cockroft; kcockroft@pennstatehealth.psu.edu
}

Received 18 November 2020; Accepted 11 January 2021; Published 19 January 2021

Academic Editor: Alessandro Stecco

Copyright (C) 2021 Cesar Velasco et al. This is an open access article distributed under the Creative Commons Attribution License, which permits unrestricted use, distribution, and reproduction in any medium, provided the original work is properly cited.

\begin{abstract}
Introduction. Many reports have described a decrease in the numbers of patients seeking medical attention for typical emergencies during the COVID-19 pandemic. These reports primarily relate to urban areas with widespread community transmission. The impact of COVID-19 on nonurban areas with minimal community transmission is less well understood. Methods. Using a prospectively maintained prehospital quality improvement database, we reviewed our hospital EMS transports with a diagnosis of stroke from January to April 2019 (baseline) and January to April 2020 (pandemic). We compared the volume of patients, transport/presentation times, severity of presenting symptoms, and final diagnosis. Results. In January, February, March, and April 2019, 10, 11, 17, and 19 patients, respectively, were transported in comparison to 19, 14, 10, and 8 during the same months in 2020. From January through April 2019, there was a 53\% increase in transports, compared to a $42 \%$ decrease during the same months in 2020, constituting significantly different trend-line slopes $(3.30$; 95\% CI $0.48-6.12$ versus -3.70 ; $95 \%$ CI -5.76--1.64, $p=0.001)$. Patient demographics, comorbidities, and symptom severity were mostly similar over the two time periods, and the number of patients with a final diagnosis of stroke was also similar. However, the median interval from EMS dispatch to ED arrival for patients with a final diagnosis of stroke was significantly longer in January to April 2020 $(50 \pm 11.7 \mathrm{~min})$ compared to the same time period in $2019(42 \pm 8.2 \mathrm{~min}, p=0.01)$. Discussion/Conclusion. Our data indicate a decrease in patient transport volumes and longer intervals to EMS activation for suspected stroke care. These results suggest that even in a nonurban location without widespread community transmission, patients may be delaying or avoiding care for severe illnesses such as stroke. Clinicians and public health officials should not ignore the potential impact of pandemic-like illnesses even in areas of relatively low disease prevalence.
\end{abstract}

\section{Introduction}

In early 2020, the COVID-19 pandemic paralyzed many urban health systems across the United States and the world. Reports suggested a drop in emergencies including heart attack and stroke were due to individuals choosing to stay at home until their symptoms worsened rather than risk hospital exposure to the coronavirus [1]. According to physicians at urban medical centers, emergency departments were seeing fifty percent of the usual number of stroke cases and reporting lower than usual census in the intensive care units that typically managed this population [2].

Unlike urban areas, the impact of COVID-19 on nonurban hospitals with minimal community transmission has been less well understood or investigated. However, the same stress of social distancing, isolation, and reluctance to seek 
care for a stroke may place nonurban patients at similar risk of poor outcomes. In this study, we examined the impact of the COVID-19 pandemic on acute stroke care at a nonurban medical center located in an area without widespread community transmission.

\section{Methods}

We retrospectively reviewed prospective data from our institution's prehospital quality improvement database and crossmatched it against data entered in the hospital's Get With The Guidelines (GWTG) Stroke Center database. Emergency Medical Systems (EMS) transports with a diagnosis of stroke were compared from January to April 2019 (baseline) and January to April 2020 (pandemic). Variables of interest from the prehospital database included the volume of patients, the interval time between last known well (LKW) and time of EMS dispatch, prehospital transport and transfer times, severity of presenting symptoms, and final clinical diagnosis. Demographic data, as well as initial stroke severity and clinical outcomes, were obtained from the institution's GWTG Stroke Center database.

This research was conducted ethically under accepted guidelines and was approved by our facility's Human Subjects Protection Office and Institutional Review Board (Study 00015437). Deidentified data used in this project will be made available to other investigators upon reasonable request to the corresponding author. This project was supported by departmental funds.

2.1. Statistical Analysis. Data were analyzed using GraphPad Prism (version 8.2.1; San Diego, CA). Univariate betweengroup comparisons were calculated using Fisher's exact test for categorical data and unpaired $t$-tests for interval data. The Mann-Whitney $U$ test was used for nonparametric ordinal data (e.g., NIHSS) and nonparametric continuous data (e.g., EMS time parameters). ANCOVA testing was utilized to compare the slopes of the linear regression lines. A $p$ value of $<0.05$ was considered significant.

\section{Results}

In the months of January, February, March, and April in 2020, the height of the COVID-19 pandemic in our region, $19,14,10$, and 8 patients, respectively, were transported by our hospital EMS with a presumed diagnosis of stroke. In comparison, during the corresponding four months in $2019,10,11,17$, and 19 patients, respectively, were transported. This represents a $53 \%$ increase in transports for January through April of 2019, compared to a $42 \%$ decrease during the same months in 2020 (Figure 1). The slopes of these trend-lines are significantly different $(3.30 ; 95 \% \mathrm{CI}$ $0.48-6.12$ versus -3.70 ; 95\% CI $-5.76--1.64, p=0.001)$. For the study period in 2019,23 of 57 total patients transported were discharged with a final diagnosis of stroke compared to 21 of 51 patients in 2020. With the exception of a higher percentage of patients with diabetes in the pre-COVID 2019 period, there were no significant differences in demographics, comorbidities, or symptom severity between the

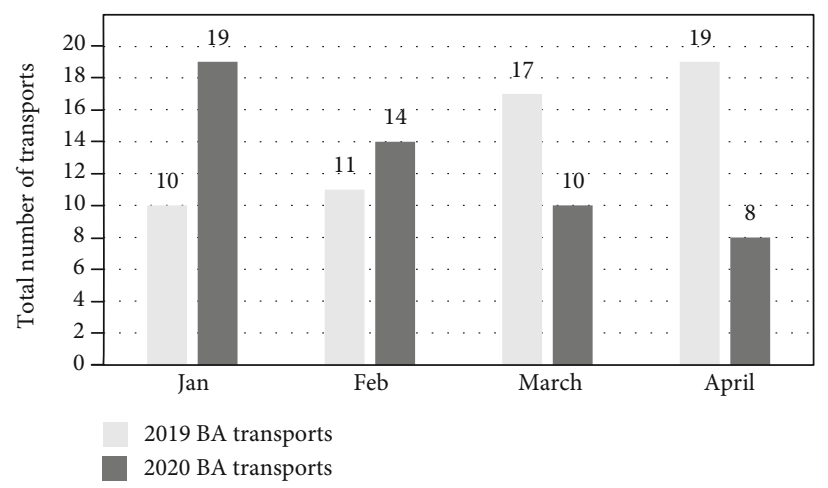

FIgURE 1: Total number of suspected stroke patients (aka brain attacks; BA) transported by EMS.

two time segments for either the entire group of patients or those found to have a final diagnosis of stroke (Table 1). An examination of EMS time metrics found the median time on the scene for EMS was shorter in January through April of 2020 versus the same months in $2019(14+5.9$ versus 17 +6.2 minutes, $p=0.028$ ) (Table 2). However, despite this, the median interval from EMS dispatch to ED arrival for patients with a final diagnosis of stroke was significantly longer in January to April 2020 (50 $\pm 11.7 \mathrm{~min})$ compared to the same time period in $2019(42 \pm 8.2 \mathrm{~min}, p=0.01)$ (Table 2). For the most part, patients from the two time periods were similar in the initial stroke evaluation, acute management, and outcomes (Table 3). Patients in pre-COVID time period were more likely to have a positive EMS stroke screening tool, but no differences were seen in the location from which patients came or in their presenting NIHSS. A similar percentage of patients received IV thrombolysis, and door to needle times were similar. Short-term outcomes were also similar in terms of discharge NIHSS and discharge disposition.

\section{Discussion}

We examined the impact of the COVID-19 pandemic on our nonurban population. Prior publications have mostly focused on the impact of the pandemic on large urban centers where disease prevalence is relatively high and community transmission tends to be more widespread [3-6]. In the 12-county region of south-central Pennsylvania where our medical center is located, there were 335 COVID-19 cases in March and 4,652 in April (https://www.health.pa.gov/topics/disease/ coronavirus/Pages/March-Archive.aspx, https://www.health .pa.gov/topics/disease/coronavirus/Pages/April-Archive.aspx) out of a population of approximately 2.1 million (approximately 10 to 221 per 100,000$)$. In comparison, in the fivecounty Philadelphia metropolitan area with a population of approximately 4 million, there were 2,550 and 24,545 cases during the same two months, respectively (approximately 64 to 614 per 100,000) (https://www.health.pa.gov/topics/disease/ coronavirus/Pages/March-Archive.aspx, https://www.health.pa .gov/topics/disease/coronavirus/Pages/April-Archive.aspx). In April, in New York City, one of the worst-hit areas in the United States, it was estimated by state officials that just over 
TABLE 1: Demographics of all patients and patients with a final diagnosis of stroke.

\begin{tabular}{|c|c|c|c|c|c|c|}
\hline & \multicolumn{3}{|c|}{ All patients } & \multicolumn{3}{|c|}{ Stroke patients } \\
\hline & 2019 (pre-COVID-19) & 2020 (COVID-19 era) & $p$ value & 2019 (pre-COVID-19) & 2020 (COVID-19 era) & $p$ value \\
\hline Age (SD) & $75.96(13.2)$ & $73.24(17.3)$ & 0.36 & $77.91(12.6)$ & $75.90(18.9)$ & 0.68 \\
\hline Female & $N(63.2 \%)$ & $N(56.9 \%)$ & 0.56 & $56.5 \%$ & $43.5 \%$ & 0.55 \\
\hline Prior stroke/TIA & & & & $41.7 \%$ & $58.3 \%$ & 0.48 \\
\hline Hypertension & & & & $79.0 \%$ & $76.5 \%$ & $>0.99$ \\
\hline Afib/flutter & & & & $15.8 \%$ & $29.4 \%$ & 0.43 \\
\hline $\mathrm{CAD} /$ prior $\mathrm{MI}$ & & & & $36.8 \%$ & $17.6 \%$ & 0.27 \\
\hline Carotid stenosis & & & & 0 & 0 & $>0.99$ \\
\hline Diabetes mellitus & & & & $47.4 \%$ & $11.8 \%$ & 0.03 \\
\hline Tobacco abuse & & & & $15.8 \%$ & $5.9 \%$ & 0.61 \\
\hline Drug/alcohol abuse & & & & $10.5 \%$ & $0 \%$ & 0.49 \\
\hline Dyslipidemia & & & & $73.7 \%$ & $58.8 \%$ & 0.48 \\
\hline Family history of stroke & & & & $5.3 \%$ & $23.5 \%$ & 0.17 \\
\hline
\end{tabular}

Demographic characteristics of patients pre- and post-COVID era presented as means and standard deviation for interval data and proportions for categorical data. Statistical significance for univariate between-group comparisons was calculated using Fisher's exact test for categorical data and unpaired $t$-tests for interval data.

TABLE 2: EMS time parameters for all patients and patients with a final diagnosis of stroke.

\begin{tabular}{|c|c|c|c|c|c|c|}
\hline & \multicolumn{3}{|c|}{ All patients } & \multicolumn{3}{|c|}{ Stroke patients } \\
\hline & $\begin{array}{c}2019 \text { (pre-COVID- } \\
19 \text { ) }\end{array}$ & $\begin{array}{l}2020 \text { (COVID-19 } \\
\text { era) }\end{array}$ & $p$ value & $\begin{array}{c}2019 \text { (pre-COVID- } \\
19 \text { ) }\end{array}$ & $\begin{array}{c}2020 \text { (COVID-19 } \\
\text { era) }\end{array}$ & $p$ value \\
\hline $\begin{array}{l}\text { LKW to dispatch (median, } \\
\text { IQR) }\end{array}$ & $67(19-347)$ & $126(12-310)$ & $p=0.86$ & $70(23-441)$ & $126(11.5-291.5)$ & $p=0.94$ \\
\hline Time on scene (median, IQR) & $16(11.5-21)$ & $14(10-17)$ & $p<0.04$ & $17(11.5-21.25)$ & $14(13-17.5)$ & $p=0.48$ \\
\hline $\begin{array}{l}\text { Dispatch to ED (median, } \\
\text { IQR) }\end{array}$ & $45(34-50.5)$ & $45(39-54)$ & $p=0.19$ & $44.5(34-49)$ & $52(42-59)$ & $p<0.03$ \\
\hline
\end{tabular}

All times in minutes. LKW: last known well; ED: emergency department. Mann-Whitney U test was used to compare EMS time parameters.

one-fifth of its residents had contracted COVID-19 [7]. Despite the relatively low disease prevalence in south-central Pennsylvania, we were concerned that people were inappropriately delaying emergency care out of fear of contracting the virus. Our present work appears to confirm this hypothesis. We found a significant decrease in the number of suspected stroke patient transports between January and April 2020, the peak of the pandemic in our region, versus the same months in 2019. These results suggest that even in nonurban areas with relatively low disease prevalence and community transmission, patients may be experiencing the same reticence in coming to the hospital. Unsurprisingly, given the widespread dissemination of COVID-19 information, the same fears and concerns present in areas of widespread community prevalence and transmission are also likely to be present in areas where infections are less common. Interestingly, there was no difference in the stroke severity at initial presentation or the rate of initial treatment with an intravenous thrombolytic. However, there was a trend toward more patients having a positive result on an EMS stroke screening tool in 2019 compared to 2020. Our examination of EMS time metrics indicated that overall time from dispatch to ED was increased in the first few months of 2020 compared to 2019, despite a slight decrease in the time on-scene. With the widely reported lack of traffic at this time, the reasons for this increase are unclear. Perhaps, concerns over appropriate personal protective equipment (PPE) may have led to some delays. Fortunately, discharge outcomes were similar, although the study was not powered to examine clinical outcomes.

The overwhelming fear of contracting COVID-19 may lead many patients to be reluctant to seek timely assistance for life-threatening emergencies, such as stroke [2, 8]. Although the practices of social distancing, sheltering in place, and self-quarantine are deemed effective in limiting the spread of the virus, these measures may also serve to discourage the pursuit of acute medical attention for nonCOVID-related diseases [9]. Patient anxiety, depression, and concern for individual safety have all been cited as potential reasons for not seeking timely treatment [9]. Additionally, the observance of stay-at-home orders may have decreased the opportunity for friends and or family members to witness a patient exhibiting stroke symptoms in a timely manner [5]. Obviously, seeking immediate evaluation and treatment for stroke is imperative since the lasting consequences of delay can include long-term disability or death [10]. This study serves to reinforce the importance of stroke 
TABLE 3: Stroke evaluation, management, and outcome.

\begin{tabular}{|c|c|c|c|}
\hline & 2019 (pre-COVID-19) & 2020 (COVID-19 era) & $p$ value \\
\hline \multicolumn{4}{|l|}{ Presenting location } \\
\hline Residence & 27 & 27 & \\
\hline Business & 10 & 4 & 0.24 \\
\hline Healthcare & 19 & 18 & \\
\hline Facility & 1 & 4 & \\
\hline \multicolumn{4}{|l|}{ Other } \\
\hline Positive EMS stroke scale & $33 / 57(57.9 \%)$ & $19 / 51(37.2 \%)$ & 0.05 \\
\hline NIHSS on admission (median, range) & $4(0-25)$ & $3(0-24)$ & 0.59 \\
\hline IV thrombolytic in ED & $5 / 22(22.7 \%)$ & $4 / 21(19.0 \%)$ & 0.99 \\
\hline Door to needle (minutes) ${ }^{\dagger}$ & 40.8 & 51.8 & 0.52 \\
\hline Stroke diagnosis confirmed & $22 / 57$ & $21 / 51$ & 0.84 \\
\hline \multicolumn{4}{|l|}{ Stroke type } \\
\hline Ischemic & 15 & 10 & \\
\hline TIA & 3 & 6 & 0.20 \\
\hline Hemorrhagic & 4 & 5 & \\
\hline NIHSS at discharge (median, range) & $3(0-36)$ & $1(0-30)$ & 0.43 \\
\hline \multicolumn{4}{|l|}{ Discharge disposition } \\
\hline Home/rehab & 44 & 35 & 0.39 \\
\hline Other & 13 & 16 & \\
\hline
\end{tabular}

EMS: Emergency Medical Services; NIHSS: National Institutes of Health Stroke Scale; IV: intravenous; ED: emergency department; TIA: transient ischemic attack. ${ }^{\dagger}$ Reported only for those patients who received IV thrombolytics. Chi-square or Fisher's exact test for categorical variables. ${ }^{*}$ Chi-square test for each variable within the EMS Stroke Scale. Mann-Whitney $U$ test for nonparametric ordinal data (i.e., NIHSS).

awareness education in the setting of a pandemic, even in areas where the pandemic may not have a profound, direct clinical impact.

Limitations to this study include the use of data associated with a single comprehensive stroke center; this is reflected in the relatively small number of suspected stroke cases analyzed between both time periods. Although data was prospectively acquired, this work involved a retrospective review and therefore is subject to the inherent biases of such studies. Although considered "nonurban" with significant rural areas, south-central Pennsylvania does include multiple towns and small cities where disease prevalence and community transmission rates may vary.

\section{Conclusion}

Our research suggests that nonurban communities with a low prevalence of COVID-19 and experiencing minimal community transmission would benefit from more public health education on the importance of seeking immediate care for acute stroke. Helping patients better understand the risks associated with avoiding medical treatment during a pandemic may help reduce the long-term financial and societal burden of stroke. As many areas prepare for second and third waves of infection, clinicians and public health officials need to be cognizant of the potential impact of the COVID-19 pandemic and other pandemic-like diseases on emergency care, even in areas where the direct effects of the pandemic may be less severe.

\section{Data Availability}

Deidentified data used in this project will be made available to other investigators upon reasonable request to the corresponding author.

\section{Conflicts of Interest}

The authors have no conflicts of interest to declare with regard to this project. However, the authors wish to disclose the following relationships with industry (RWI): Cesar Velasco-none; Brandon Wattai-none; Scott Buchle-none; Alicia Richardson-none; Varun Padmanaban-none; Kathy J. Morrison-none; Ray Reichwein-Athersys, Inc. (grant); Ephraim Church-none. Scott D. Simon-none; and Kevin M. Cockroft-Medtronic Neurovascular (consultant), Actuated Medical (consultant, shareholder), Minnetronix (consultant), Nico Corporation (grant), Intersocietal Accreditation Commission (Board of Directors, grant), and NeuroPoint Alliance (Board of Directors).

\section{Authors' Contributions}

Cesar Velasco contributed to the conceptualization, investigation, formal analysis, and writing (original draft). Brandon Wattai contributed to the investigation and data curation. Scott Buchle contributed to the investigation and data curation. Alicia Richardson contributed to the conceptualization and writing (reviewing and editing). Varun Padmanaban contributed to the formal analysis and writing (reviewing 
and editing). Kathy J. Morrison contributed to the conceptualization and writing (reviewing and editing). Ray Reichwein contributed to the conceptualization and writing (reviewing and editing). Ephraim Church contributed to the writing (reviewing and editing). Scott D Simon contributed to the writing (reviewing and editing). Kevin M. Cockroft contributed to the conceptualization, methodology, writing (reviewing and editing), and supervision.

\section{References}

[1] H. M. Krumholz, "Where Have all the Heart Attacks Gone?," https://www.nytimes.com/2020/04/06/well/live/coronavirusdoctors-hospitals-emergency-care-heart-attack-stroke.html.

[2] G. Kolata, "A heart attack? NO, it was the coronavirus," https://www.nytimes.com/2020/03/27/health/coronaviruscardiac-heart-attacks.html.

[3] S. Rudilosso, C. Laredo, V. Vera et al., "Acute stroke care is at risk in the era of COVID-19: experience at a comprehensive stroke center in Barcelona," Stroke, vol. 51, no. 7, pp. 19911995, 2020.

[4] R. Pop, V. Quenardelle, A. Hasiu et al., "Impact of the COVID19 outbreak on acute stroke pathways - insights from the Alsace region in France," European Journal of Neurology, vol. 27, no. 9, pp. 1783-1787, 2020.

[5] J. Zhao, H. Li, D. Kung, M. Fisher, Y. Shen, and R. Liu, "Impact of the COVID-19 epidemic on stroke: care and potential solutions," Stroke, vol. 51, no. 7, pp. 1996-2001, 2020.

[6] T. J. Oxley, J. Mocco, S. Majidi et al., "Large-vessel stroke as a presenting feature of Covid-19 in the young," The New England Journal of Medicine, vol. 382, no. 20, 2020.

[7] NPR, "Coronavirus has infected a 5th of New York City, testing suggests".

[8] H. S. Markus and M. Brainin, "COVID-19 and stroke-a global World Stroke Organization perspective," International journal of stroke, vol. 15, no. 4, pp. 361-364, 2020.

[9] C. M. Schirmer, A. J. Ringer, A. S. Arthur et al., "Delayed presentation of acute ischemic strokes during the COVID-19 crisis," Journal of Neuro Interventional Surgery, vol. 12, pp. 639942, 2020.

[10] W. J. Powers, A. A. Rabinstein, T. Ackerson et al., “2018 Guidelines for the early management of patients with acute ischemic stroke: a guideline for healthcare professionals from the American Heart Association/American Stroke Association," Stroke, vol. 49, no. 3, pp. e46-e110, 2018. 